\title{
Robust Control for Helicopters Performance Improvement: an LMI Approach
}

\author{
Luiz Ricardo Trajano da Silva ${ }^{1, *}$, Victor Augusto Fernandes de Campos ${ }^{1}$, Alain Segundo Potts ${ }^{1}$
}

How to cite

\author{
Silva LRT (D) https://orcid.org/0000-0003-2157-9608 \\ Campos VAF (D https: / / orcid.org/0000-0003-4193-6289 \\ Potts AS (D) https: / / orcid.org/0000-0002-8130-6268
}

\begin{abstract}
Silva LRT, Campos VAF, Potts AS (2020) Robust Control for Helicopters Performance Improvement: an LMI Approach. J Aerosp Tecnol Manag, 12 e3620. https://doi.org/10.5028/ jatm.v12.1179
\end{abstract}

ABSTRACT: This paper presents an LMI (Linear Matrix Inequalities) application for the design of robust controllers for multivariate systems that have multiple points of operation. Some systems change their parameters along time, then, it is necessary to switch the control for different operational points. The purpose of this controller is to ensure the stability and performance requirements of the system for different operating points with the same controller. The method uses the following concepts of predefined structures controller, LMI region, and polytopic systems. To validate the controller a linearized model of a helicopter was used. These helicopters belong to a system class of MIMO (Multiple-Input Multiple Outputs) type and present a complex dynamic in their flight modes, therefore, due to these features, this type of helicopter is a good model to implement and test the efficiency of the described method in this work. The results were satisfactory. Some limitations in its implementation were found and discussed. An LQG (Linear-Quadratic-Gaussian) controller was also designed for the same model of the helicopter just for comparison. Analyzing the settling time properties, the LMI controller presented a better response than the LQG controller.

KEYWORDS: Linear Matrix Inequalities; Region pole placement; Robust control; Helicopter; Polytopic system.

\section{INTRODUCTION}

The many robust linear control techniques are part of a broad area within the theory of control systems. These control techniques operate under certain conditions where it is not possible to accurately guarantee the parameters of the real plant model, as well as the influence of external perturbations on the dynamics of the system.

Through the years, various techniques and tools have been developed for the design of robust controllers, which allows the combined use of these tools to create other or new techniques, therefore, this work uses a set of tools together with the aim to develop a robust controller for a helicopter under several operational points. The proposed robust controller has to stabilize the plant and improve the damping response for all these points with the same controller.

The controller presented in this work is developed using the pole placement in LMI regions with the concept of polytopic systems.

The pole placement in LMI regions is a technique that places the system closed-loop poles in certain regions on the left half-plane of the complex plane, employing for that, the solution of a set of LMIs, where LMI means Linear Matrix Inequalities. The LMI is a great tool for solving optimization problems, it allows the multi-objective controllers design; that is, the controller designed satisfies both the stability and the performance requirements of the control system, and this is one of its advantages (Andrade et al., 2017). Great work about LMI can be seen in Scherer et al. (1997) where it explores in depth several possibilities of using the LMI for controlling problems, including, beyond the LMI regions, controls that use $H_{\infty}$ and $H_{2}$ norms among others. In Chilali et al. (1999) the concept of pole placement in regions using LMIs is approached, where the main aim of this concept is to improve the system damping response.

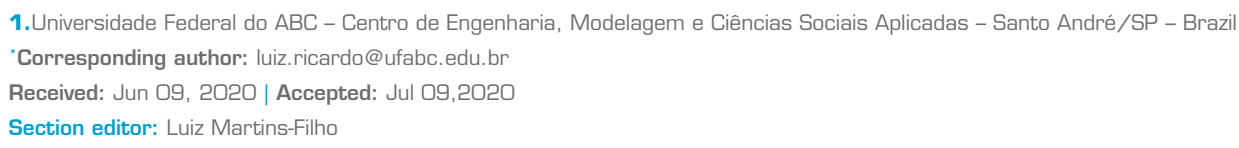


The polytopic system is used to improve the robustness of the controller, and as such, the controller is designed to operate under various operational points.

A helicopter plant offers a great model to analyze the efficiency of the robust control techniques, because it is a complex system, multivariable, with dynamics highly coupled and nonlinear. Therefore, there are some aspects that must be considered in controller design, including mainly the robustness and stability.

There are many studies about robust control for helicopters and in most of these studies, the techniques that used $H_{\infty}$ and $H_{2}$ controls are presented. We can cite a work where a comparison was made between $H_{\infty}$ and $H_{2}$ techniques for helicopter position control via radio. Both control techniques were implemented, and its results were taken in separate. Then, the work concluded that the cost benefit of using the $H_{2}$ technique was better than using $H_{\infty}$ (Weilenmann et al., 1994).

Another approach using $H_{\infty}$ technique for helicopter control can be seen in (Yoneyama and Kikuchi, 2002). The work develops a relationship between quadratic stability and $H_{\infty}$ control problem for uncertain systems, with the aim to design a controller for quadratic stabilization of uncertain system using $H_{\infty}$ controllers. The developed controller was implemented in a helicopter model HUMUSOFT CE 150. This helicopter belongs to systems of MIMO (Multi-Input Multi-Output) type, with two inputs and two outputs. Posteriorly, the results of the implemented controller were compared with an optimal controller in order to verify its effectiveness.

Models with less complexity were also studied. A Helicopter attitude control with two DoF (Degrees of Freedom) was also analyzed (Sakamoto et al., 2006). This work disregards the coupled dynamics modes of the helicopter. For attitude control, PID controllers were implemented in separate, one PID controller for elevation and other for yaw. The results show that this approach is capable of stabilizing the system and fulfill the tracking signal requirements.

The LQR control technique is also used to design controls for helicopters. It can be seen in Shen et al. (2009), a control law based in LQR (Linear Quadratic Regulator) used to stabilize the three Euler angles of the helicopter. In order to verify the technique efficiently, simulations in HIL (Hardware In the Loop) were done. The HIL simulation type is broadly used in research steps for engineering systems. It is a non-destructive simulation that provides safety, money, and time saving, as well as facilitating data collection.

The attitude control problem of pitch and elevation channels with three DoF is shown in (Wang et al., 2013). In this study, a controller is designed with the aim to improve system performance, tracking signal response for a unit step input, and get a robust stabilization for parameter uncertain and external perturbations. The dynamics models of pitch and elevation channels which were considered uncoupled became two SISO (Single-Input Single-Output) systems with various uncertainties, for example, nonlinearity, non-modeled dynamics, among others. An $H_{\infty}$ controller was used, separately, for each SISO channel. A robust compensator was used to improve the closed-loop system robustness. The experimental results showed the robustness improvement for the closed-loop system.

In Kaway et al. (2017), a robust control problem for a 3 DoF Helicopter was presented. A set of models to be controlled was generated by a linearization of the model in finite points. For each one of these linearized models a controller based on $H_{\infty}$ and $\mathrm{H}_{2}$ was designed. The problem formulated to controller allows it to be stated as an LMI problem, thus a set of LMIs was solved to get the controller. The simulation results showed the efficiency of the implemented controller.

Lastly, in Razzaghian (2018) a neural network based on adaptive control was proposed to a miniature unmanned helicopter. The helicopter model is approximate to a neural network with radial base function (RBF) and its adaptation laws were developed using Lyapunov stability theory. The work presents a sliding mode method that uses a neural network based on adaptative control. The results of this sliding model were compared with the classic sliding model control with the same problem in order to analyze its effectiveness. The simulation results showed the success of the implementation.

Through the literature review, it has been seen that both the ways of using LMIs and the use of helicopter models to test new control methodologies remain current. Therefore, this work is proposed to implement pole allocation in regions using LMIs in conjunction with the concept of polytopic systems for stabilization of a helicopter and its improvement over the initial conditions.

This paper is organized as follows: firstly the work will present the helicopter dynamic model in state space form and its general characteristics, what is obtained mostly from (Padfield, 2008). Then, in an introductory way, the LMI regions concept will be 


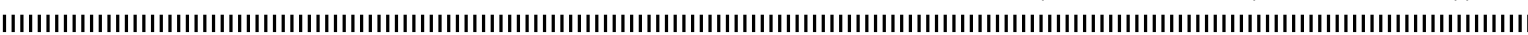

shown. The work continues with the mathematical formulation of the controller structure for the system, showing the structure of pre-defined controller and its transfer functions. Finally, the method to get the controller and its robust procedure will be shown. The results of the tests and simulations of the implemented controller to a helicopter Westland Lynx and its comparison to an LQG controller will be shown and discussed at the end of this work.

\section{HELICOPTER MODEL}

The proposed design to the robust controller shown in this work will be implemented over a linearized helicopter model of Westland Lynx Mk7 for six operation points. It is a research helicopter used by Britain Defense Research Agency (DRA), which is available in (Padfield, 2008).

The main components that describe the helicopter dynamics for six DoF are shown in Fig. 1 . Where $X, Y$ and $Z$ represent the external aerodynamical forces acting along x-, y- and z-axes, $L_{h}, M_{h}$ and $N_{h}$ represent the external aerodynamic moments around the $\mathrm{x}-, \mathrm{y}$ - and $\mathrm{z}$-axes, $u, v$ and $w$ represent the translational velocity components of helicopter along fuselage $\mathrm{x}-, \mathrm{y}-\mathrm{and} \mathrm{z}$-axes, $p$, $q$ and $r$ represent the angular velocities $(\mathrm{rad} / \mathrm{s})$ around fuselage $x$-, $y$ - and $z$-axes, and $c g$ means center of gravity.

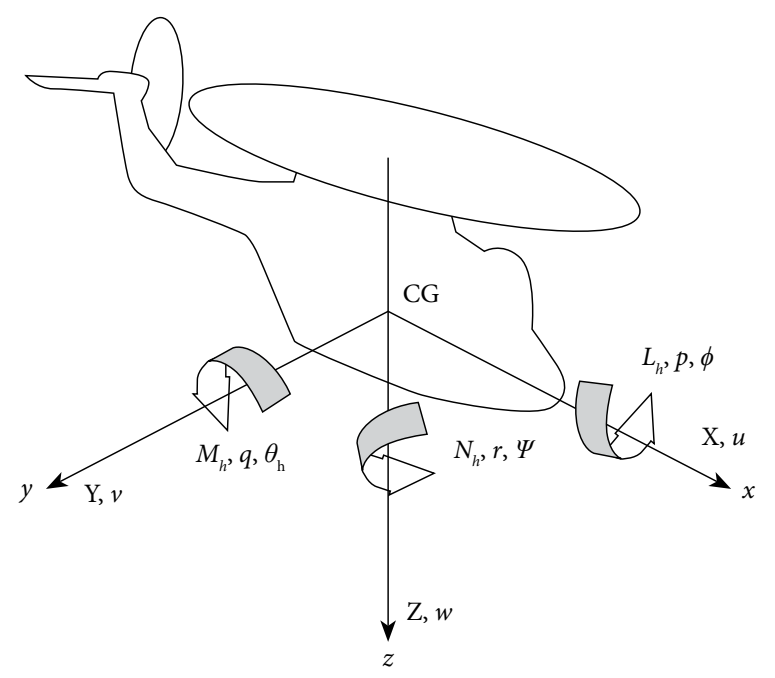

Figure 1. Helicopter orthogonal axes system.

The motion equations for a rigid body helicopter for six DoF, can be described as follows (Luo et al., 2003):

Force Equations:

$$
\begin{aligned}
\dot{u} & =r v-q w+\frac{X}{m_{s}}-g \sin \theta_{h} \\
\dot{v} & =p w-r u+\frac{Y}{m_{s}}+g \cos \theta_{h} \sin \phi \\
\dot{w} & =q u-p v+\frac{Z}{m_{s}}+g \cos \theta_{h} \cos \phi
\end{aligned}
$$

Moment Equations:

$$
\begin{aligned}
\dot{p} & =\left(c_{1} r+c_{2} p\right) q+c_{3} L+c_{4} N \\
\dot{q} & =c_{5} p r-c_{6}\left(p^{2}-r^{2}\right)+c_{7} M \\
\dot{r} & =\left(c_{8} p-c_{2} r\right) q+c_{4} L+c_{9} N
\end{aligned}
$$


Attitude Equations:

$$
\begin{aligned}
\dot{\phi} & =p+(q \sin \phi+r \cos \phi) \tan \theta_{h} \\
\dot{\theta} & =q \cos \phi-r \sin \phi \\
\dot{\psi} & =\frac{q \sin \phi+r \cos \phi}{\cos \theta_{h}}
\end{aligned}
$$

where the moment equations coefficients are defined as:

$$
\begin{array}{ll}
c_{1}=\frac{\left(I_{y y}-I_{z z}\right) I_{z z}-I_{x z}^{2}}{\Gamma} & c_{6}=\frac{I_{x z}}{I_{y y}} \\
c_{2}=\frac{\left(I_{x x}-I_{y y}+I_{z z}\right) I_{x z}}{\Gamma} & c_{7}=\frac{1}{I_{y y}} \\
c_{3}=\frac{I_{z z}}{\Gamma} & c_{8}=\frac{I_{x x}\left(I_{x x}-I_{y y}\right)+I_{x z}{ }^{2}}{\Gamma} \\
c_{4}=\frac{I_{x z}}{\Gamma} & c_{9}=\frac{I_{x x}}{\Gamma} \\
c_{5}=\frac{I_{z z}-I_{x x}}{I_{y y}} & \Gamma=I_{x x} I_{z z}-I_{x z}{ }^{2}
\end{array}
$$

The variables $I_{X X}, I_{X Z}$ and $I_{Y Y}$ are the inertial moments of the helicopter and $m_{S}$ is its total mass. The $X, Y$ and $Z$ forces and $L_{h}$, $M_{h}$ and $N_{h}$ moments are the results obtained from the sum of all forces and moments of each one of the helicopter subsystems. Then, we write:

$$
\begin{aligned}
X & =X_{R}+X_{T}+X_{F}+X_{t p}+X_{f n} \\
Y & =Y_{R}+Y_{T}+Y_{F}+Y_{t p}+Y_{f n} \\
Z & =Z_{R}+Z_{T}+Z_{F}+Z_{t p}+Z_{f n} \\
L_{h} & =L_{R}+L_{T}+L_{F}+L_{t p}+L_{f n} \\
M_{h} & =M_{R}+M_{T}+M_{F}+M_{t p}+M_{f n} \\
N_{h} & =N_{R}+N_{T}+N_{F}+N_{t p}+N_{f n}
\end{aligned}
$$

The above equations that present the notations $R, T, F, t p$ and $f n$, which means, respectively, the following subsystems: main rotor empennage, fuselage, tail plane and fin.

\section{SYSTEM INPUT}

The helicopter model used in this work has four control inputs for its operation. They are the main $\theta_{0}$ and tail $\theta_{0 T}$ collective pitch angle and the longitudinal $\theta_{1 S}$ and lateral $\theta_{1 C}$ cyclic pitch. Through main collective control $\theta_{0}$, it is possible to increase the helicopter lift force and change its vertical velocity. That means, the main collective control is used to increase or decrease the propeller angle of attack changing, therefore, the helicopter lift force.

The longitudinal $\theta_{1 S}$ and lateral $\theta_{1 C}$ cyclic controls are used to move the helicopter in all directions, controlling the pitch and roll movement.

Lastly, the tail collective control $\theta_{0 T}$ controls the tail rotor impulse force with the aim to control the helicopter yaw movements, in order to stabilize it in a desired position and auxiliary in lateral movements. This control is important to generate a counterforce to the main rotor movement and, hence, allowing the stabilization of the helicopter. 


\section{LINEARIZED MODEL}

The set of equations, Eq.(1) to Eq.(5), constitute the nonlinear equations of the helicopter dynamics, but in order to implement the linear controls techniques it is necessary the linearization of these equations. For this reason, the small perturbations theory is applied a distinct operating point of the helicopter, thus allowing the implementation of the linear control technique presented in this work. The representation in the state space of the linearized model of the helicopter presented in this work was obtained in (Padfield, 2008).

\section{STATE SPACE REPRESENTATION}

The system state space representation is given by (Ogata, 2009):

$$
\begin{aligned}
& \dot{x}=A x+B u_{i} \\
& y=C x
\end{aligned}
$$

where the $A, B$ and $C$ matrices represent the matrix of the state, the input and the output, respectively, and the vectors $x, u_{i}$ and $y$ represent the vector of the state variables, the input and the output respectively:

$$
\begin{aligned}
& x=\left[u w q \theta_{h} v p \phi r\right] \\
& u_{i}=\left[\theta_{0} \theta_{1 s} \theta_{1 c} \theta_{0 T}\right]
\end{aligned}
$$

In the state vector $x$, the variables $u, v$ and $w$ represent the translational velocities, the variables $p, q$ and $r$ represent the angular velocities and the variables $\phi$ and $\theta_{h}$ represent the Euler angles.

The output vector $y$ is given by:

$$
y=C x=\left[q \theta_{h} \phi r\right]
$$

The matrix $A$ and $B$ of the state space representation for a linearized model of a helicopter, with eight state variables, are presented in (Padfield, 2008) and shown in Eq.(9) and Eq.(10):

$$
\begin{gathered}
A=\left[\begin{array}{cccc}
X_{u} & X_{w}-Q_{e} & X_{q}-W_{e} & -g \cos \Theta_{e} \\
Z_{u}+Q_{e} & Z_{w} & Z_{q}+U_{e} & -g \cos \Phi_{e} \sin \Theta_{e} \\
M_{u} & M_{w} & M_{q} & 0 \\
0 & 0 & \cos \Phi_{e} & 0 \\
Y_{u}-R_{e} & Y_{w}+P_{e} & Y_{q} & -g \sin \Phi_{e} \sin \Theta_{e} \\
L_{u}^{\prime} & L_{w}^{\prime} & L_{q}^{\prime}+k_{1} P_{e}-k_{2} R_{e} & 0 \\
0 & 0 & \sin \Phi_{e} \tan \Theta_{e} & \Omega_{a} \sec \Theta_{e} \\
N_{u}^{\prime} & N_{w}^{\prime} & N_{q}^{\prime}-k_{1} R_{e}-k_{3} P_{e} & 0 \\
X_{v}+R_{e} & X_{p} & 0 & X_{r}+V_{e} \\
Z_{v}-P_{e} & Z_{p}-V_{e} & -g \sin \Phi_{e} \cos \Theta_{e} & Z_{r} \\
M_{v} & M_{p}-2 P_{e} I_{x z} / I_{y y} & 0 & M_{r}-2 R_{e} I_{x z} / I_{y y} \\
0 & -R_{e}\left(I_{x x}-I_{z z}\right) / I_{y y} & 0 & -P_{e}\left(I_{x x}-I_{z z}\right) / I_{y y} \\
Y_{v} & 0 & -\Omega_{a} \cos \Theta_{e} & -\sin \Phi_{e} \\
L_{v}^{\prime} & Y_{p}+W_{e} & g \cos \Phi_{e} \cos \Theta_{e} & Y_{r}-U_{e} \\
0 & L_{p}^{\prime}+k_{1} Q_{e} & 0 & L_{r}^{\prime}-k_{2} Q_{e} \\
N_{v}^{\prime} & 1 & 0 & \cos \Phi_{e} \tan \Theta_{e} \\
N_{p}^{\prime}+k_{3} & 0 & N_{r}^{\prime}-k_{1} Q_{e}
\end{array}\right]
\end{gathered}
$$


The control matrix $B$ given by:

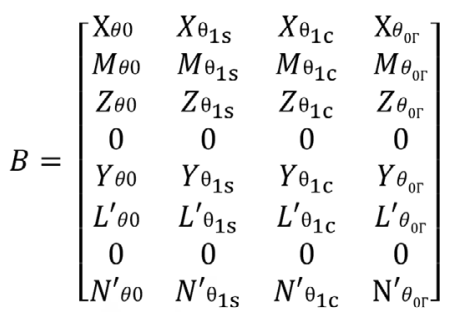

The matrix $A$ and $B$ represent the helicopter dynamic system, where each one of its elements is derivatives obtained for each operation points or flight condition.

\section{LMI REGION}

An LMI region is defined as any subset $D$ of the complex plane that can be written as:

$$
\mathcal{D}=\left\{z \in \mathbb{C}: L+z M+\bar{z} M^{T}<0\right\}
$$

where $L$ and $M$ are real matrices such that $L=L^{T}$ and $\bar{Z}$ represents the complex conjugate of $\mathrm{z}$. The matrix function it is called the characteristic function of $D$ :

$$
f_{\mathcal{D}}(z)=L+z M+\bar{z} M^{T}
$$

The main LMI regions used in pole allocation technique in control systems are:

The strip region, $R_{e}(z)<-a$

$$
f_{\mathcal{D}}(z)=z+\bar{z}+2 \alpha<0
$$

The conic sector fixed at the origin and with an internal angle of $2 \theta$ :

$$
f_{\mathcal{D}}(z)=\left[\begin{array}{ll}
\sin \theta(z+\bar{z}) & \cos \theta(z-\bar{z}) \\
\cos \theta(\bar{z}-z) & \sin \theta(z+\bar{z})
\end{array}\right]<0
$$

Some important features about the LMI regions are:

- The intersection of LMI regions is also LMI regions.

- Any region symmetrical about the real axis can be approximated by an LMI region.

- A real matrix $A$ is considered $D$-stable if it has all its eigenvalues in the LMI region and if there is only one symmetric matrix Q such that:

$$
\begin{gathered}
M_{\mathcal{D}}(A, Q)=L \otimes Q+M \otimes(A Q)+M^{T} \otimes\left(Q A^{T}\right)<0, \\
Q>0
\end{gathered}
$$

where $\otimes$ represents the product of Kronecker.

\section{CLOSED-LOOP SYSTEM AND CONTROLLER STRUCTURE}

As seen previously, the state space representation of a dynamic system is given by: 


$$
\dot{x}=A x+B u_{i}
$$

The controller used for helicopter control is of the predefined type, which allows the choice of the controller poles in advance. A representation of the closed-loop system with the controller, in regulator mode, can be seen in Fig. 2.

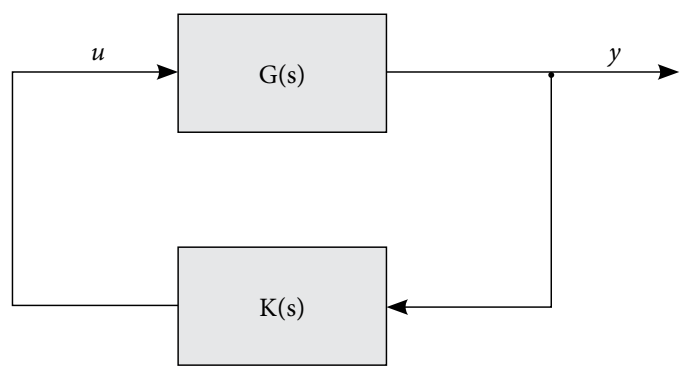

Figure 2. Closed-loop system (Regulator).

The matrix $G(s)$ and $K(s)$, respectively, represent the transfer function of the plant and the controller. The controller has a transfer function for each input/output relation. This transfer function can be written, in general, as:

$$
K_{y k \rightarrow u l}=\frac{a_{y k \rightarrow u l} \cdot s^{2}+b_{y k \rightarrow u l} \cdot s+c_{y k \rightarrow u l}}{s^{2}+\left(p_{1}+p_{2}\right) \cdot s+p_{1} \cdot p_{2}}
$$

The notation $y k \rightarrow u l$ indicates the transfer function that relates the output $y k$ with $u l$ input, where $k$ indicates the numbers of outputs and $l$ indicates the numbers of inputs. The pre-defined poles are represented by $P_{1}$ and $P_{2}$, and they are chosen freely by the designer. The controller aims to get the values of the coefficients $a_{y k \rightarrow u l}, b_{y k \rightarrow u l}$ and $c_{y k \rightarrow u l}$ so that the closed-loop poles remain within a desired region on the left half plane of the complex plane, even with the addition of perturbations in the model of the plant.

The matrix $K(\mathrm{~s})$ can also be represented in state space representation:

$$
\begin{array}{r}
\dot{x_{c}}=A_{c} x_{c}+B_{c} y \\
u=C_{c} x_{c}+D_{c} y
\end{array}
$$

where the $A_{C}, B_{C}, C_{C}$, and $D_{C}$ matrices represent the state matrix, the input matrix, the output matrix and the feedforward matrix of the controller, respectively, and the vectors $x_{c}, y$ and $u_{i}$ represent the state variables vector, the input vector and the output vector of the controller, respectively.

The matrices $A_{C}$, and $C_{C}$ are formed by following equation (Andrade et al., 2017):

$$
\begin{aligned}
A_{c} & =\left[\begin{array}{ccccc}
0 & 1 & \ldots & 0 & 0 \\
k & j & \ldots & 0 & 0 \\
\vdots & \vdots & \ddots & \vdots & \vdots \\
0 & 0 & \ldots & 0 & 1 \\
0 & 0 & \ldots & k & j
\end{array}\right] \\
C_{c} & =\left[\begin{array}{ccccc}
0 & 1 & \ldots & 0 & 0 \\
\vdots & \vdots & \ddots & \vdots & \vdots \\
0 & 0 & \ldots & 0 & 1
\end{array}\right]
\end{aligned}
$$

where $k=-P_{1} \cdot P_{2}$ and $j=\left(P_{1}+P_{2}\right)$. Thus, the matrices $A_{C}$ and $C_{C}$ are known as the pre-defined matrices of the controller.

The closed-loop system, Fig. 2, can be represented in state space form using Eq.(6) and Eq.(14), obtaining the following equation: 


$$
\left[\begin{array}{c}
\dot{x} \\
\dot{x_{c}}
\end{array}\right]=\left[\begin{array}{cc}
A+B \cdot D_{C} \cdot C & B \cdot C_{c} \\
B_{c} \cdot C & A_{c}
\end{array}\right] \cdot\left[\begin{array}{c}
x \\
x_{c}
\end{array}\right]
$$

In order to use the LMI tool for the development of the controller it is also necessary to make a new representation of the dynamic system, where:

$$
\begin{aligned}
A_{m} & =\left[\begin{array}{cc}
A & B \cdot C_{c} \\
0 & A_{c}
\end{array}\right], B_{m}=\left[\begin{array}{ll}
B & 0 \\
0 & I
\end{array}\right] \\
C_{m} & =\left[\begin{array}{ll}
C_{m} & 0
\end{array}\right]
\end{aligned}
$$

Thus, the controller gain matrix is given by:

$$
K_{c}=\left[\begin{array}{l}
D_{c} \\
B_{c}
\end{array}\right]
$$

With this, the representation of the dynamic system becomes:

$$
\begin{aligned}
\dot{x}_{m} & =A_{m} x_{m}+B_{m} u_{m} \\
y & =C_{m} x_{m}
\end{aligned}
$$

where $x_{m}=\left[\begin{array}{ll}x & x_{c}\end{array}\right]^{T}$ and the state feedback control law is given by um $=K_{c} \cdot y=K_{c} \cdot C_{m} \cdot x_{m}$.

Thus, using Eq. (17), the values of the matrices $A_{m}, B_{m}$ and $C_{m}$ are obtained. The problem then becomes to find the matrix $K_{C^{\prime}}$ which gives us the values of the unknown matrices $D_{C}$ and $B_{C}$. With the values of the matrices $D_{C}$ and $B_{C}$ it is possible to fully describe the controller dynamic, Eq.(14), and find the coefficients values $a_{y k \rightarrow u l}, b_{y k \rightarrow u l}$ and $c_{y k \rightarrow u l}$ in such a way that the transfer function of each relation between inputs and outputs of the controller $k_{y k \rightarrow u l}$ Eq.(13), is determined.

\section{ROBUST CONTROLLER DESIGN}

\section{STATE FEEDBACK CONTROL}

The objective of the proposed controller is to use the feedback of the system states for the allocation of closed-loop poles in a certain region of the complex plane. This region is defined in a way that the allocated poles have the desired damping value for improving system performance.

The region of the complex plane chosen for the controller design lies in the left half-plane, obviously, and is defined by the intersection of two LMI regions. The LMI regions used in the present study are semi-plane $\mathbb{H}_{\alpha}$ and conical sector $\mathbb{D}_{\theta}$, represented by Fig. 3 .

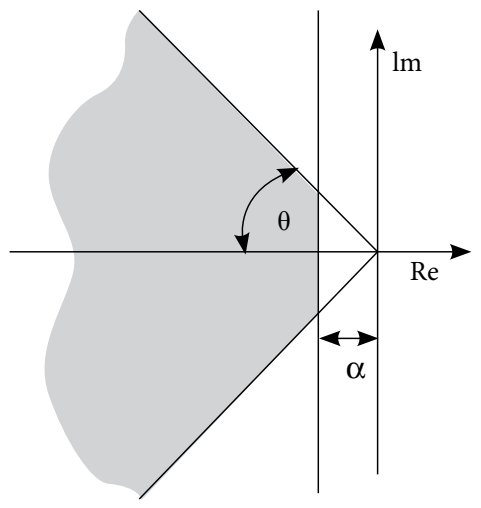

Figure 3. The intersection of the LMI region for pole allocation. 
The $\mathbb{H}_{\alpha}$ region is defined by:

$$
\begin{aligned}
\mathbb{H}_{\alpha} & =\{x+j y \mid x<-\alpha\} \\
& =\left\{z \mid \mathcal{R}_{e}(z)<-\alpha\right\} \\
& =\left\{z \mid \frac{1}{2}(z+\bar{z})<-\alpha\right\}
\end{aligned}
$$

Thus, the $\mathbb{H}_{\alpha}$ is an LMI region that has:

$$
L=2 \alpha \text { and } M=1 \text {. }
$$

The conical sector $\mathbb{D}_{\theta}$ is defined by:

$$
\begin{aligned}
\mathbb{D}_{\theta} & =\{x+j y|| y \mid<-x \tan \theta\} \\
& =\left\{x+j y \mid y^{2}<x^{2} \tan ^{2} \theta, \quad x \tan \theta<0\right\} \\
& =\left\{x+j y \mid y^{2} \cos \theta<x^{2} \sin ^{2} \theta, \quad x \sin \theta<0\right\} \\
& =\left\{x+j y \mid\left[\begin{array}{cc}
x \sin \theta & i y \cos \theta \\
-i y \cos \theta & x \sin \theta
\end{array}\right]<0\right\} \\
& =\left\{z \mid\left[\begin{array}{cc}
(z+\bar{z}) \sin \theta & (z-\bar{z}) \cos \theta \\
(-z+\bar{z}) \cos \theta & (z+\bar{z}) \sin \theta
\end{array}\right]<0\right\}
\end{aligned}
$$

Thus, the $\mathbb{D}_{\theta}$ is an LMI region that has:

$$
L=0 \text { and } M=\left[\begin{array}{cc}
\sin \theta & \cos \theta \\
-\cos \theta & \sin \theta
\end{array}\right]
$$

The closed-loop system representation, Eq.(19), according to control law $u_{m}=K_{c} \cdot y$ is written as:

$$
\begin{aligned}
\dot{x}_{m} & =A_{m} x_{m}+B_{m} u_{m}=A_{m} x_{m}+B_{m} K_{c} \cdot y \\
& =\left(A_{m}+B_{m} K_{c} C_{m}\right) x_{m}=A_{c l} x_{m}
\end{aligned}
$$

Substituting matrix $A$ for the matrix $A_{c l}$ in Eq.(12), the term $A_{c l} Q$ must be represented as follows:

$$
A_{c l} Q=A_{m} Q+B_{m} \cdot K_{c} \cdot C_{m} \cdot Q
$$

In order to solve the problem of finding the value of the matrix $K_{C}$ in a problem that can be solved through the LMI, it is necessary firstly, to perform the following variable changes:

$$
K_{C} \cdot C_{m} \cdot Q=N \cdot C_{m}
$$

Substituting Eq.(26) into Eq.(25):

$$
A_{c l} Q=A_{m} Q+B_{m} \cdot N \cdot C_{m}
$$

Thus, the LMI solution will provide us with the values of the matrix variables $Q$ and $N$. 
Posteriorly, to obtain the value of $K_{C}$, it is necessary to calculate the value of matrix $M$ :

$$
\begin{aligned}
M \cdot C_{m} & =N \cdot C_{m} \\
M & =C_{m} \cdot Q \cdot C_{m}^{T} \cdot\left(C_{m} \cdot C_{m}^{T}\right)^{-1}
\end{aligned}
$$

Then, using equations Eq.(26) and Eq.(28) we arrive at the equation to obtain the $K_{C}$ :

$$
\begin{aligned}
K_{c} \cdot M \cdot C_{m} & =N \cdot C_{m} \\
K_{c} & =N \cdot M^{-1}
\end{aligned}
$$

Therefore, the problem is related to finding the value of the matrix variables $Q$ and $N$ that allow the recovery of the matrix $K_{C}$ through the set of equations Eq.(28) and Eq.(29). Using the latter results in conjunction with Eq.(12) we obtain a new representation of the LMI region, Eq. (30), which, when solved, will give us the values of the $Q$ and $N$ matrices:

$$
L \otimes Q+M \otimes\left(A_{m} Q+B_{m} \cdot N \cdot C_{m}\right)+M^{T} \otimes\left(Q \cdot A_{m}^{T}+C_{m}^{T} \cdot N^{T} \cdot B_{m}^{T}\right)<0
$$

By solving Eq. (30) simultaneously for the two LMI regions we will obtain a matrix $K_{C}$ which would ensure that the poles of the closed-loop system are located in the region determined by those regions in the complex plane.

Correctly replacing the values of $L$ and $M$ of Eq.(21) in Eq.(30) for a region of the half plan, we obtain:

$$
2 \alpha Q+\left(A_{m} Q+B_{m} \cdot N \cdot C_{m}\right)+\left(Q \cdot A_{m}^{T}+C_{m}^{T} \cdot N^{T} \cdot B_{m}^{T}\right)<0
$$

Similarly, for a region of the conic sector type, replacing the values of $L$ and $M$ of Eq.(23) in Eq.(30) the following LMI is valid:

$$
\left[\begin{array}{ll}
a A_{c l} Q+a Q A_{c l}^{T} & b A_{c l} Q-b Q A_{c l}^{T} \\
b Q A_{c l}^{T}-b A_{c l} Q & a A_{c l} Q+a Q A_{c l}^{T}
\end{array}\right]<0
$$

where:

$$
\begin{gathered}
A_{c l} Q=A_{m} Q+B_{m} \cdot N \cdot C_{m} \\
Q A_{c l}^{T}=Q \cdot A_{m}^{T}+C_{m}^{T} \cdot N^{T} \cdot B_{m}^{T} \\
a=\sin \theta \\
b=\cos \theta
\end{gathered}
$$

\section{ROBUST PROCEDURE AND POLYTOPIC SYSTEMS}

The robust procedure seeks to ensure that the designed controller maintains system performance for several different operating points. In order to do this, it is necessary to introduce the topic of polytopic systems.

The definition of polytopic systems used in $\mathrm{Gu}$ et al. (2014) is presented below. A polytopic system is a linear time-variant system represented by: 


$$
\begin{aligned}
& \dot{x}(t)=A(t) x+B(t) u \\
& y(t)=C(t) x+D(t) u
\end{aligned}
$$

This system can also be represented using the following notation:

$$
S(t)=\left[\begin{array}{ll}
A(t) & B(t) \\
C(t) & D(t)
\end{array}\right]
$$

The system, Eq.(35), varies within a fixed set of possible matrices, represented by the set $\Omega$, where:

$$
\Omega=\sum_{i=1}^{q} \alpha_{i} S_{i}: \alpha_{i} \geq 0, \sum_{i=1}^{q} \alpha_{i} S_{i}=1
$$

In the above equation the values of $S 1, S 2, \ldots, S q$ are known as vertices of the system:

$$
S_{i}=\left[\begin{array}{cc}
A_{i} & B_{i} \\
C_{i} & D_{i}
\end{array}\right], i=1, \ldots, q
$$

In this way, the polytopic system $S(t)$ is a linear combination of the matrix system $S 1, \ldots, S q$, for example, $S(t)=\alpha 1 S 1+\ldots+\alpha q S q$. Where $\alpha 1, \ldots, \alpha q$ are called polytopic coordinates of $S(t)$.

Using the concept of polytopic systems, we can rewrite Eq.(17) and Eq.(27) as follows:

$$
\begin{gathered}
A_{m, i}=\left[\begin{array}{cc}
A_{i} & B_{i} \cdot C_{c} \\
0 & A_{c}
\end{array}\right], B_{m, i}=\left[\begin{array}{cc}
B_{i} & 0 \\
0 & I
\end{array}\right] \\
A_{c l, i} Q=A_{m, i} Q+B_{m, i} \cdot N \cdot C_{m}
\end{gathered}
$$

The value of $i$ represents the operating point of the system, which can range from $1, \ldots, q$, where $q$ is the total number of operating points.

Using the results of Eq.(38) and Eq.(39) we can rewrite the linear matrix inequalities Eq.(31) and Eq.(32) as:

$$
2 \alpha Q+\left(A_{m, i} Q+B_{m, i} \cdot N \cdot C_{m}\right)+\left(Q \cdot A_{m, i}^{T}+C_{m}^{T} \cdot N^{T} \cdot B_{m, i}^{T}\right)<0
$$

Recalling that $\alpha$ delimits the maximum value of the negative real part that a pole can have

For a region of the conical sector type, the following LMI is valid:

$$
\left[\begin{array}{ll}
a A_{c l, i} Q+a Q A_{c l, i}^{T} & b A_{c l, i} Q-b Q A_{c l, i}^{T} \\
b Q A_{c l, i}^{T}-b A_{c l, i} Q & a A_{c l, i} Q+a Q A_{c l, i}^{T}
\end{array}\right]<0
$$


Since $Q$ is a positive definite matrix, the following linear matrix inequality must be included in the set of linear matrix inequalities that make up the problem:

$$
Q>0
$$

\section{METHOD SYNTHESIS}

The procedure for obtaining the robust controller for $q$ operating points is described below:

1. Solve the linear matrix Inequalities Eq.(40), Eq.(41) and Eq.(42) simultaneously for each operating point $i$, in order to obtain the value of the matrix variables $N$ and $Q$.

2. Use the result of $Q$ in conjunction with Eq.(28) to obtain the value of $M$.

3. Use the result of $M$ and $N$ together with Eq.(29) to obtain the value of $K c$.

4. Use Eq.(18) to obtain the $B_{C}$ and $D_{C}$.

5. Using the value of the state space matrices $A_{C^{\prime}} B_{C}, C_{C}$ and $D_{C^{\prime}}$ which represent the dynamics of the robust controller, obtaining the controller transfer function for each input-output relationship and consequently the controller coefficients $a_{y k \rightarrow u l^{p}} b_{y k \rightarrow u l}$ and $c_{y k \rightarrow u l}$.

\section{RESULTS}

The robust procedure described in the previous section was implemented on the linearized real size model of the Lynx Helicopter for six operating points, as Table 1 . The values of the matrices $A$ and $B$ that compose the state space representation for each one of the operating points are available in (Padfield, 2008). When the method is being applied, thirteen LMI's are generated with two matrix variables, $N$ and $Q$, which must be solved simultaneously. In this study, the LMIs were solved using the robust control toolbox of Matlab ${ }^{\circledast}$ software (Gahinet et al., 1994). The controller designed was able to stabilize the plant and improve the system's damping response. To validate the results of the implemented controller, an LQG controller was also designed for each one of the operating points.

Table 1. Operating point

\section{Operating point}

2

4

5

6

\section{Flight mode}

Hover

Forward flight 20 Knots

Forward flight 40 Knots

Forward flight 60 Knots

Forward flight 80 Knots

Forward flight 100 Knots

\section{PRE-DEFINED CONTROLLER}

The project variables of the controller are the poles $P_{1}$ and $P_{2}$, and the variables $\alpha$ and $\theta$, that are related to the LMI region. The $\alpha$ value had its value fixed in -0.005 , because during the implementation, values of $\alpha$ less than -1 made the formulated LMI problem infeasible. Following the definitions described in Campos et al. (2006) and in Andrade et al. (2017) the value of the pole $P_{1}$ will be equal to the pole $P_{2}$. To define the values of the $P_{1,2}$ and $\theta$ the robust procedure was implemented for a set of values. To do this, four angles and thirty-five possible poles were chosen for the controller design, as can be seen in equations Eq. (43) and Eq. (44): 


$$
\theta=\left[45^{\circ} 35^{\circ} 25^{\circ} 15^{\circ}\right]
$$

$$
\begin{aligned}
& p_{1,2}=[-5-10-15-20-25-30-35-40-45-50 \\
& -55-60-65-70-75-80-85-90-95-100-150 \\
& -200-250-300-350-400-450-500-600-700 \\
& -800-900-1000-1500-2000]
\end{aligned}
$$

During the tests, it was verified that the best results were obtained when the poles of the predefined controller were between -10 and -50 , so the poles that were chosen for the construction of the predefined controller were $P_{1,2}=-40$ and for the region of the conic sector the value of $\theta=25^{\circ}$. The value of $\theta$ is related to the minimum value of the damping factor allowed for the poles of the closed-loop system, with the damping factor given by the relation in Eq. (45) (Ogata, 2009):

$$
\zeta=\cos \theta=\cos (25) \cong 0.906
$$

Initially, Fig. 4 shows all the poles of the open loop systems for each of the six operating points. It is possible to see that the linearized model of the helicopter has poles in the right half-plane for all operating points, indicating that the system is nominally unstable. Besides that analyzing Fig. 4, it can be verified that several poles of the open loop systems have a damping factor lower than that proposed by the method of this study, the method proposes minimum damping of 0.906 , while the majority of the poles of the open loop system is lower than 0.3 .

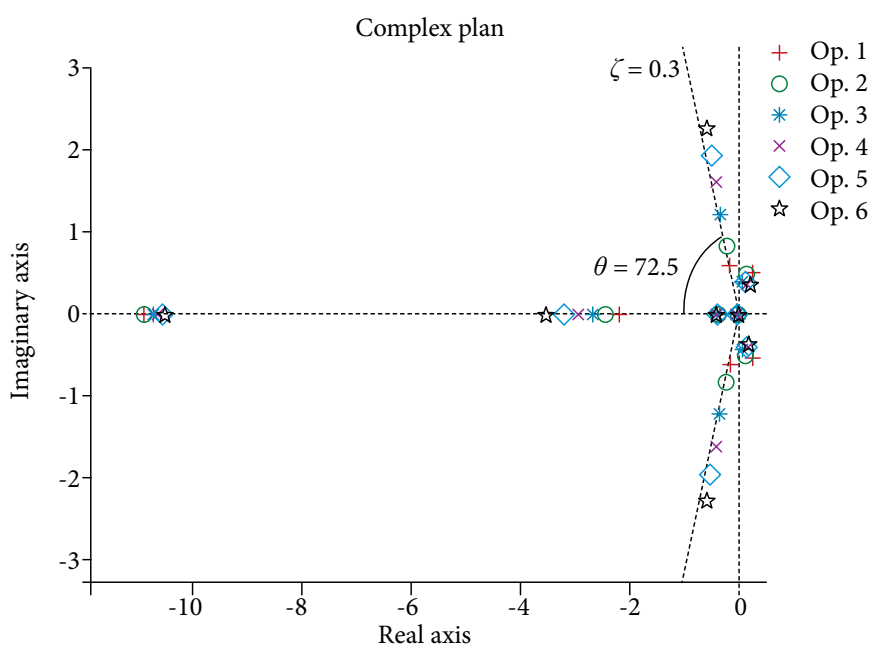

Figure 4. Open-loop system poles.

Figure 5 shows the poles of the system after the implementation of the method. As desired, it was discovered that all poles of the closed-loop system were allocated within the aimed region for each one of the operating points.

Figure 6 and Fig. 7 are an enlarged image and closer to the origin for a better view of the poles of the system within the LMI region. The lowest value obtained (absolute value) for one negative pole in the closed-loop system was 0.01 , which happened at operation point 2 , arriving close to the limit value of 0.005 , which corresponds to the value of $\alpha$. The lowest damping factor was 0.975 , in the operating point 6 , well above the limit of 0.906 . 


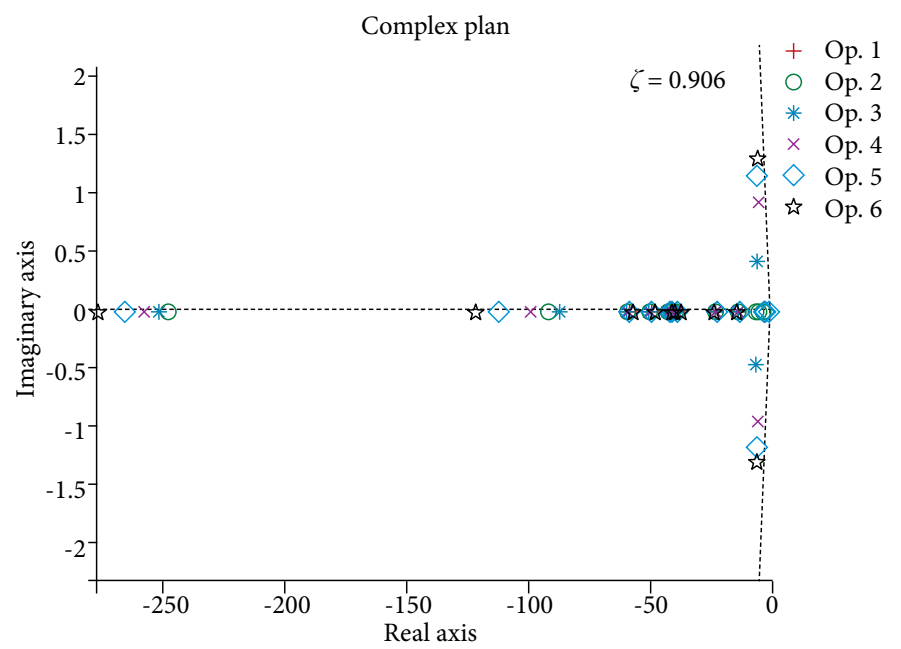

Figure 5. Closed loop system poles - LMI controller.

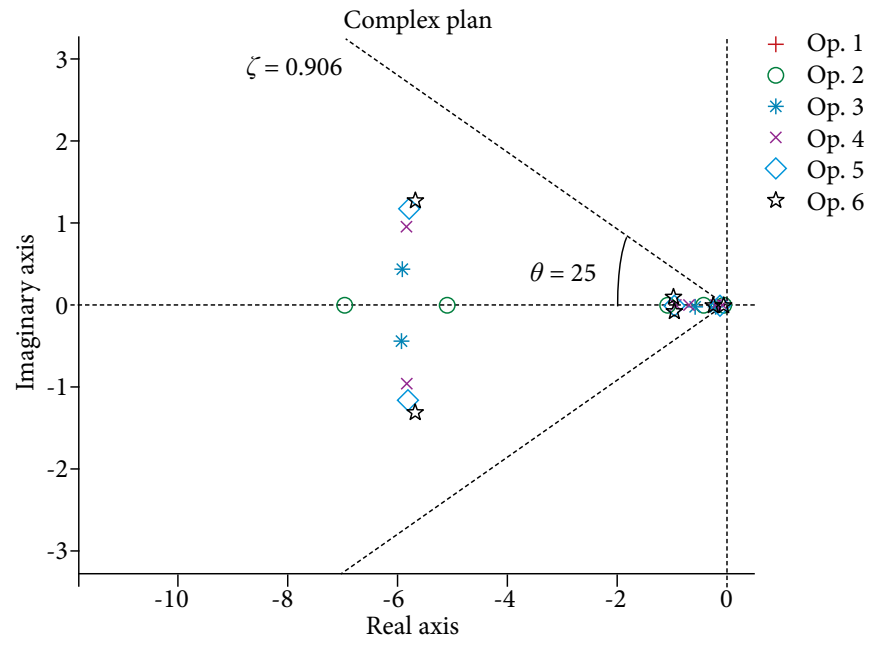

Figure 6. Closed loop system poles - LMI - (Close to origin. From 0 to -12).

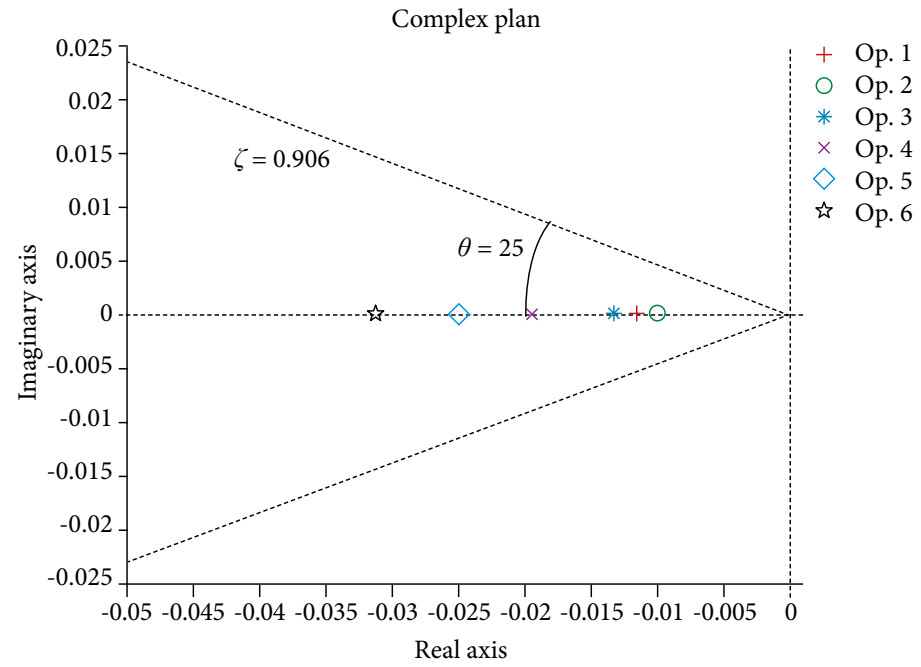

Figure 7. Closed loop system poles - LMI - (Close to origin. From 0 to -0.05 ). 
The coefficients $a_{y k \rightarrow u l}, b_{y k \rightarrow u l}$ and $c_{y k \rightarrow u l}$ of the controller, which were missing to obtain the transfer function of the Eq.(13) was obtained. The values of the coefficients can be visualized in Table 2 .

Table 2. Controller Coefficients

\begin{tabular}{|c|c|c|c|c|}
\hline$y$ & $\mu_{\mathrm{i}}$ & $a_{y k} \rightarrow_{u l}$ & $b_{y k} \rightarrow_{u l}$ & $C_{y k} \rightarrow_{u l}$ \\
\hline \multirow{4}{*}{$q$} & $\theta_{0}$ & 0.08617 & 32.18 & 798.8 \\
\hline & $\theta_{1 S}$ & 0.7162 & 57.46 & 665 \\
\hline & $\theta_{1 C}$ & -0.04339 & -3.511 & -72.77 \\
\hline & $\theta_{0 T}$ & -0.09209 & -2.088 & 99.57 \\
\hline \multirow{4}{*}{$\theta$} & $\theta_{0}$ & -8.143 & -533.2 & -6341 \\
\hline & $\theta_{1 S}$ & -9.112 & -600 & -6880 \\
\hline & $\theta_{1 C}$ & 0.4448 & 31.37 & 490.4 \\
\hline & $\theta_{0 T}$ & -0.8717 & -61.72 & -1131 \\
\hline \multirow{4}{*}{$\phi$} & $\theta_{0}$ & 1.69 & 114.3 & 1357 \\
\hline & $\theta_{1 S}$ & 1.906 & 127.5 & 1380 \\
\hline & $\theta_{1 C}$ & 0.2254 & 15.8 & 239.6 \\
\hline & $\theta_{0 T}$ & 0.2197 & 16.28 & 174.8 \\
\hline \multirow{4}{*}{$r$} & $\theta_{0}$ & 3.696 & 300.2 & 6235 \\
\hline & $\theta_{1 S}$ & 2.944 & 241.5 & 5174 \\
\hline & $\theta_{1 C}$ & -0.5729 & -42.05 & -618.5 \\
\hline & $\theta_{0 T}$ & 10.24 & 798.7 & $1.43 \mathrm{e} 04$ \\
\hline
\end{tabular}

\section{LQG CONTROLLER}

An LQG controller, which is a controller widely used in the aerospace area, was designed for each operation point in order to have a comparative parameter for the response of the predefined structure controller.

The LQG controller used in this work was designed based on (Cruz, 1996). Essentially, in the LQG controller, a Kalman filter is used to get the gains of the state observer in the optimal way. From the estimated state variables by Kalman filter it is possible to get the optimal gains to controller.

It is important to note that an LMI controller is the same controller for all operating points while that a LQG controller was designed to each one of the operating points.

For the LQG controller, the penalty matrices needed to be defined. Initially, the $Q$ and $R$ matrices for the LQG controller design were defined as:

$$
\begin{gathered}
Q=C^{T} C \\
R=I_{4}
\end{gathered}
$$

After simulations, new values of these matrices were empirically defined to improve the LQG controller response: 


$$
\begin{gathered}
Q(4,4)=500 \\
R=0.1 I_{4}
\end{gathered}
$$

The LQG controller also needs the definition of the noise matrices values, then for the intensity matrix of the plant disturbance $(\Xi)$ and for the intensity matrix of the sensor noise $(\Theta)$, the following values were chosen:

$$
\begin{aligned}
& \Xi=0.1 I_{8} \\
& \Theta=0.1 I_{8}
\end{aligned}
$$

Thus, the LQG controller was implemented for each one of the operating points. The Fig. 8 shows all poles of the system after the implementation of the LQG controller for each one of the six operating points, the Fig. also shows the line that represents the damping ratio of the LMI region.

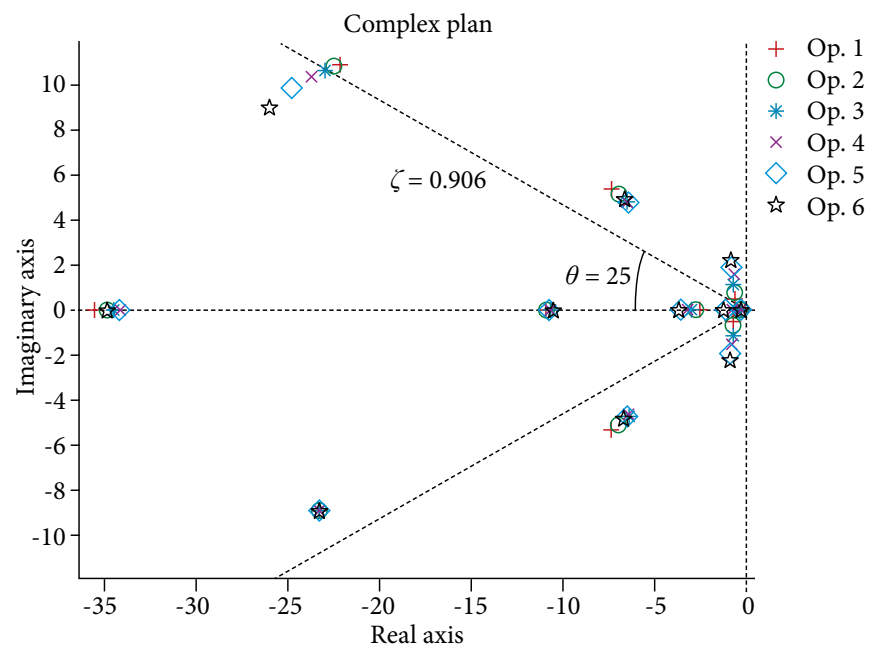

Figure 8. Closed loop system poles - LQG controller.

The Fig. 9 and Fig. 10 are an enlarged image and closer to the origin for a better view of the poles of the system within the LMI region.

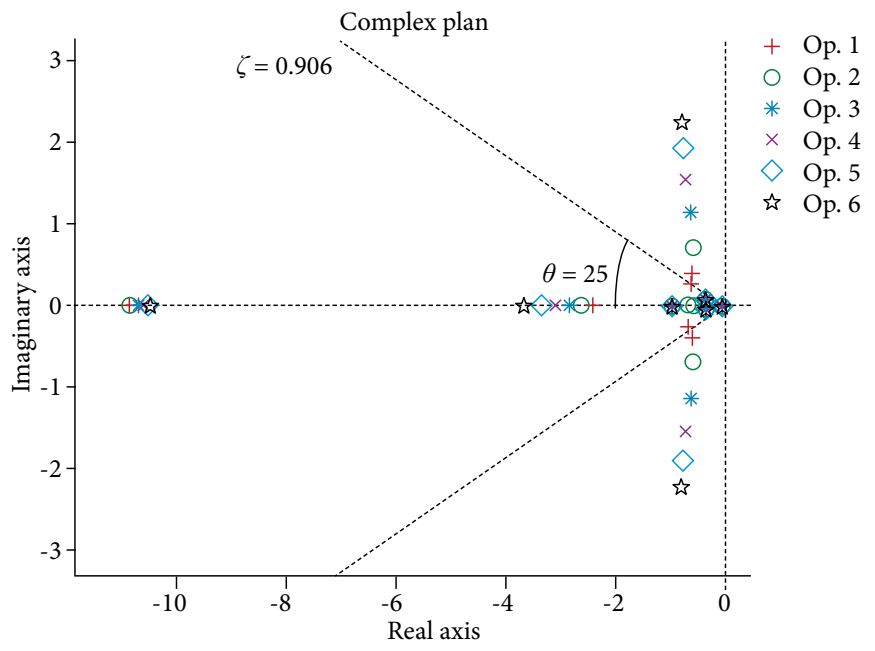

Figure 9. Closed loop system poles - LQG - (Close to origin. From 0 to -12). 


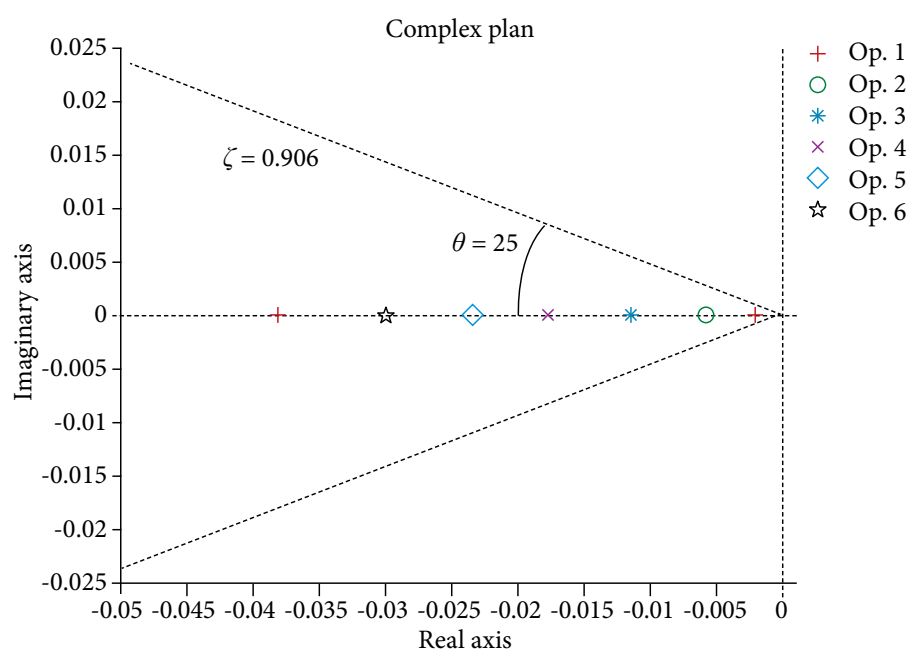

Figure 10. Closed loop system poles - LQG - (Close to origin. From 0 to -0.05).

\section{COMPARISON BETWEEN CONTROLLERS}

The proposed controller reached the objectives of stabilizing the plant and improve the system damping response for a multivariable plant for different operating points. But in order to compare its results with another controller that is known, it was simulated the initial condition response of the controllers (LMI approach and LQG), and then it was observed the ability of the closed-loop system, for each operating point, to return to its equilibrium condition.

Figure 11 and Fig. 12 show the system response to the initial conditions for the predefined structure controller and the LQG controller, respectively.

Analyzing the LQG controller response it is possible to note that the difference between response of each one operating point can be easily distinguished in Fig. 12, whereas, in the response of the predefined structure controller (LMI approach), Fig. 11, it is almost impossible to distinguish the different lines representing the response of each one operating point of the helicopter. In this first analyze, the predefined structure controller has a more uniform response during the change of the helicopter operating points.

The response of the initial conditions between the controllers was plotted for each one of the operating points separately, as we can see from Fig. 13 to Fig. 18.

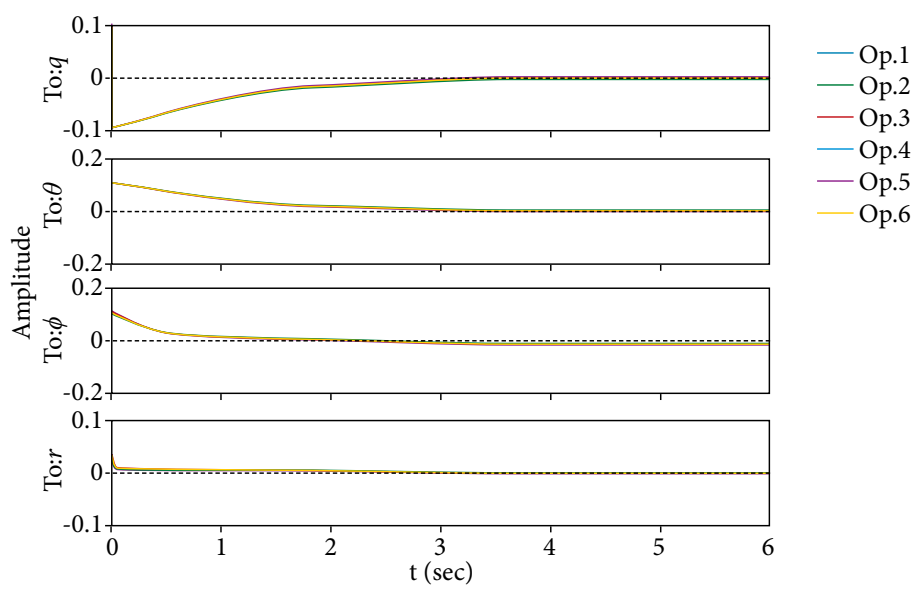

Figure 11. Response to initial conditions of the pre-defined structure controller. 


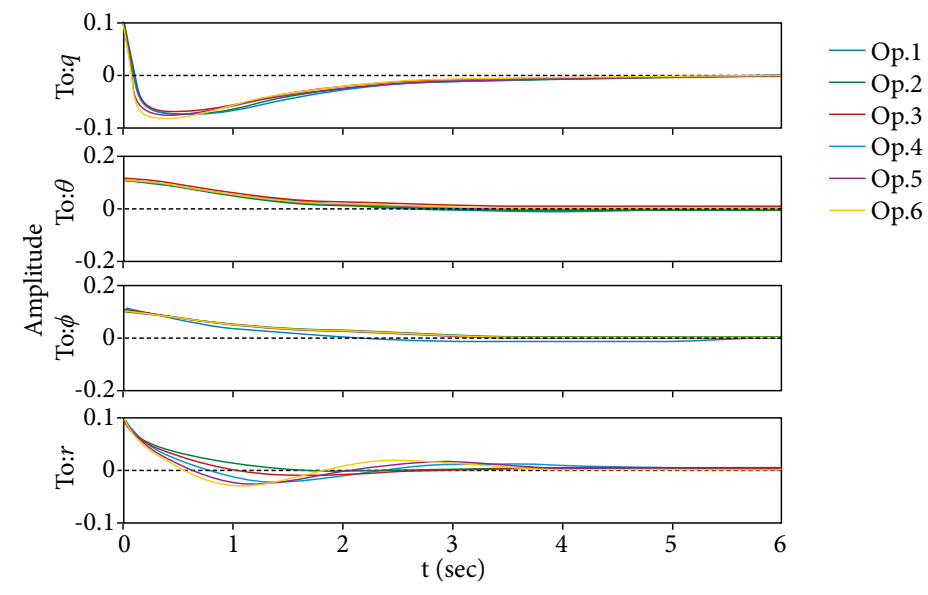

Figure 12. Response to initial conditions of the LQG controller.

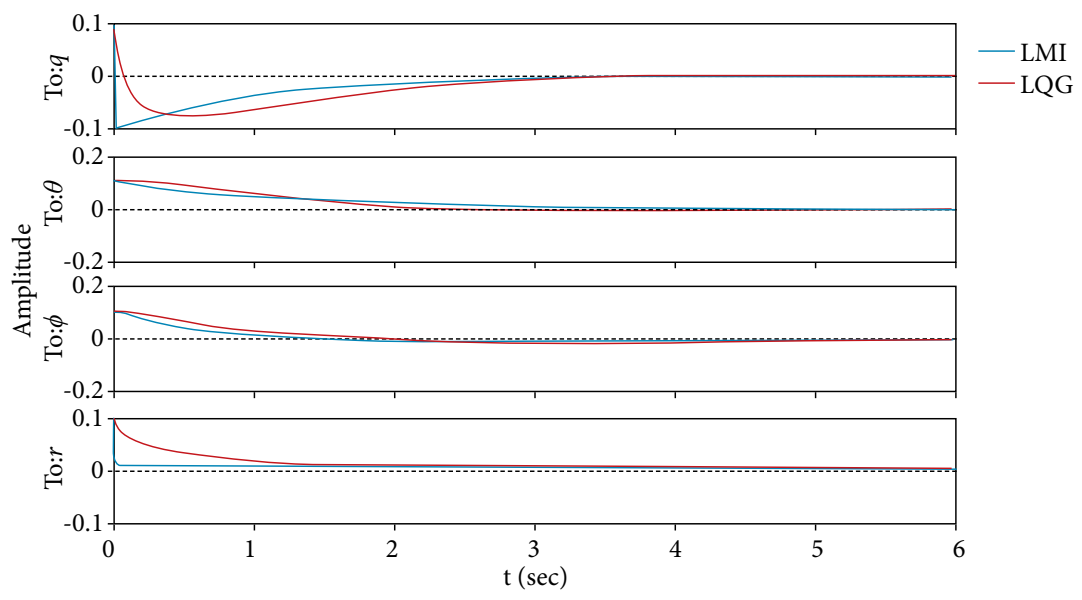

Figure 13. Comparison between controllers -Operating p. 1.

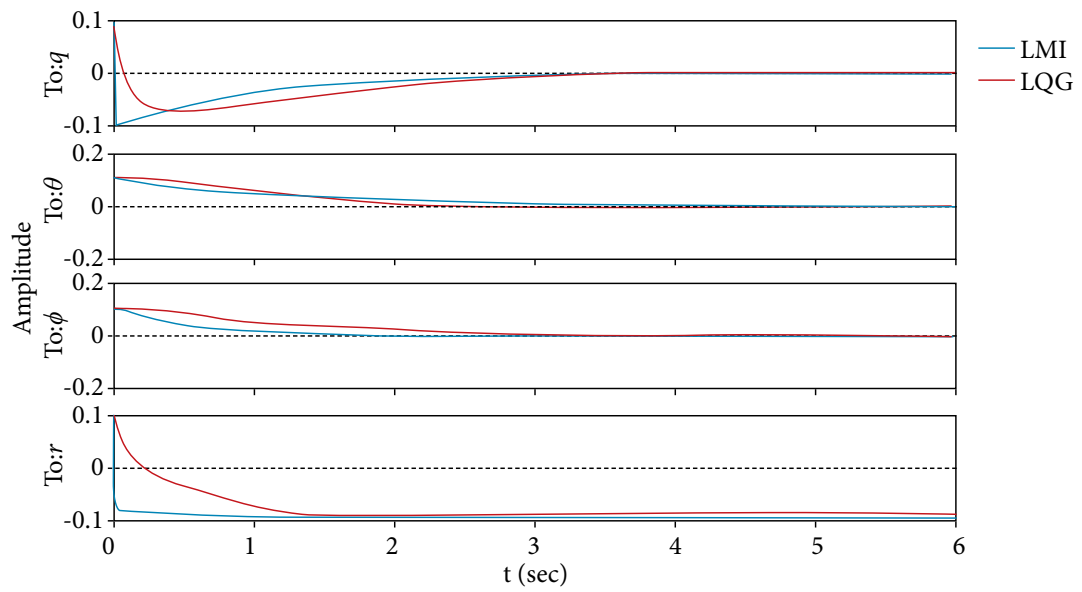

Figure 14. Comparison between controllers -Operating p. 2. 


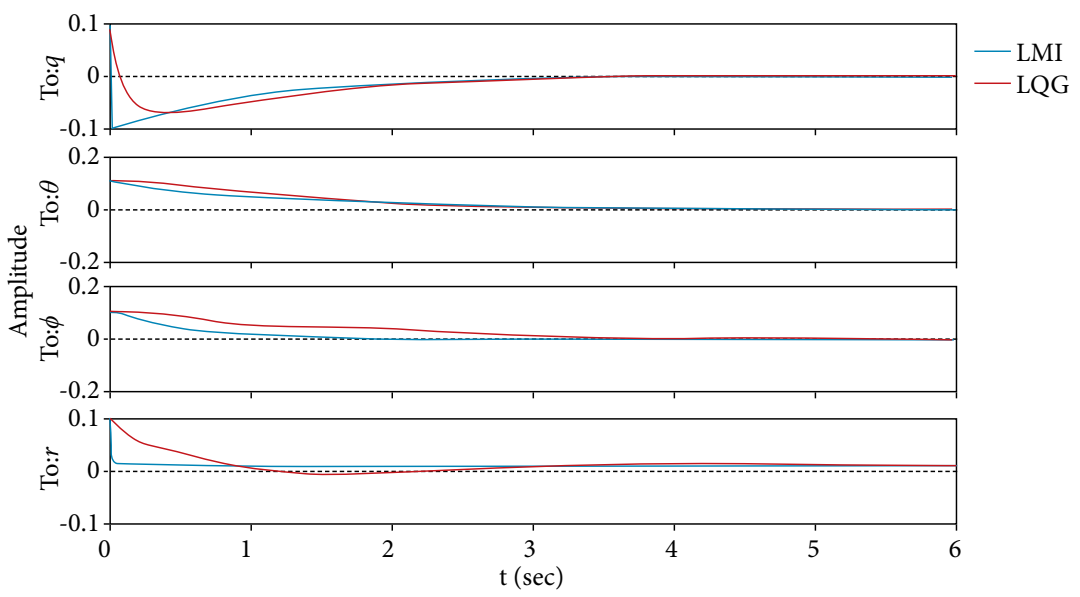

Figure 15. Comparison between controllers -Operating p. 3.

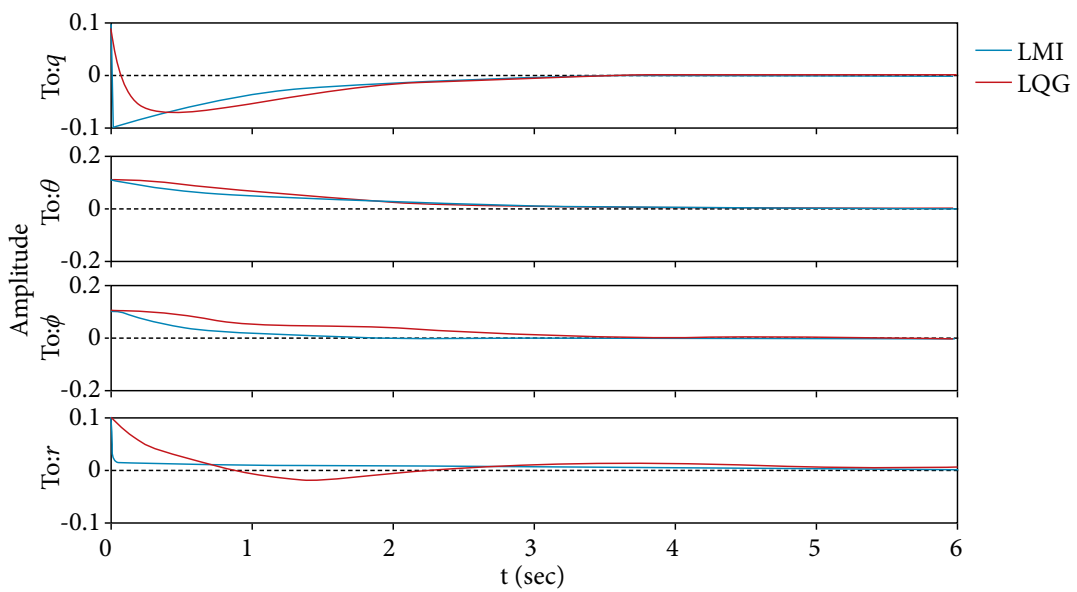

Figure 16. Comparison between controllers -Operating p. 4.

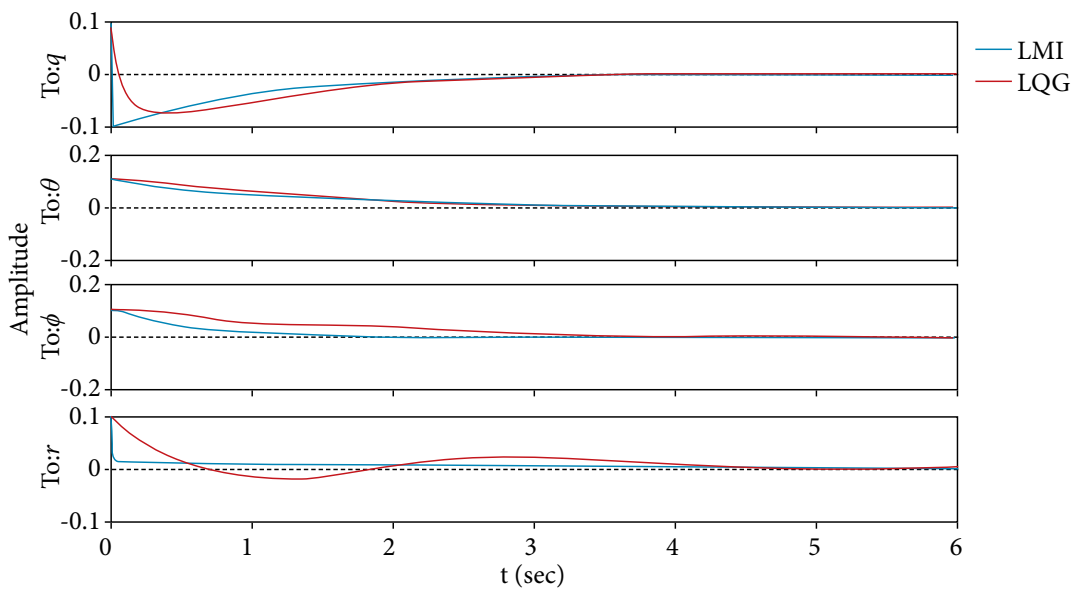

Figure 17. Comparison between controllers -Operating p. 5. 


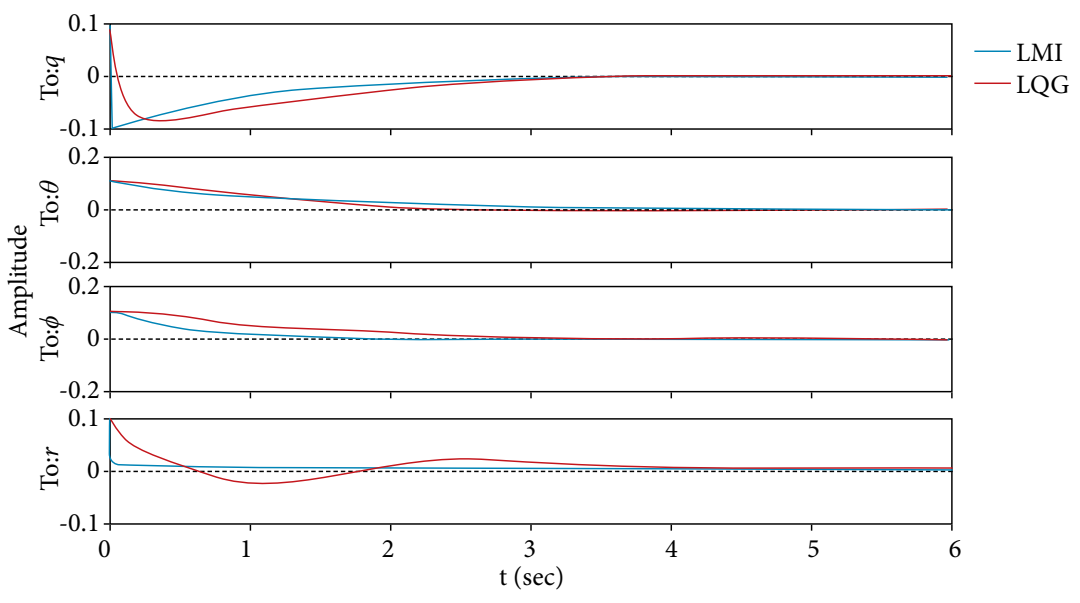

Figure 18. Comparison between controllers -Operating p. 6.

It can see from these last figures that the predefined structure controller has a similar performance when compared to an LQG control for all operating points. This shows that this method is viable for robust controller design. For the LQG controller, one controller was designed for each operating point, while just one robust controller is necessary with the predefined structure controller for all operating points. For the outputs $q$ and $\theta$ both controllers have practically the same settling time for all operating points. However, for the outputs $r$ and $\phi$ the predefined structure controller has a faster settling time, presenting a better performance than the LQG controller in this question.

\section{DISCUSSION}

This work presented the robust procedure for the design of the pre-defined structure controller with pole allocation in LMI regions and polytopic systems. A brief passage through the concept of LMI regions and the description of the procedure for obtaining the robust controller was shown. The linearized model of a helicopter for six operating points was also observed in this study.

For the chosen values of $\alpha$ and $\theta$, which determine the LMI region, the method was able to allocate the poles in the desired region, however, it was observed that there are limits when choosing these values. For other sets of values of $\alpha$ and $\theta$, the LMI solution does not converge, meaning by this, it is not always that there will be solutions for a certain region LMI. For example, $\alpha$ values greater than -1 already make the LMI solution infeasible for the problem presented in this work. The method was able to stabilize the plant and improve the system's damping response as desired.

To conclude, a comparison was made between the responses obtained by the predefined structure controller and the LQG controller. The comparison was made for each of the six operating points. The response of these controllers to an initial condition other than the equilibrium position of the system was analyzed. In the simulations that were performed, the pre-defined structure controller presented a better response than the LQG controller which was implemented, and while analyzing the settling time properties, it was shown a satisfactory result for the continuity of the studies referred to the method presented in this work.

\section{AUTHOR'S CONTRIBUTION}

Conceptualization: Silva LRT; Methodology: Silva LRT and Campos VAF; Software: Silva LRT; Original Draft: Silva LRT; Writing - Review \& Editing: Silva LRT and Campos VAF and Potts AS; Supervision: Campos VAF. 


\section{ACKNOWLEDGMENTS}

Editors and authors are thankful to Fundação Conrado Wessel for providing the financial support for publishing this article.

\section{REFERENCES}

Andrade JPP, Campos VAF, Potts AS, Garcia C (2017) Damping Improvement of a F-16 Aircraft through Linear Matrix Inequalities. IFACPapersOnLine 50(1) : 3947-3952. https://doi.org/10.1016/j.ifacol.2017.08.293

Campos VAF, Cruz JJ, Zanetta LC (2006) Pole placement and robust adjustment of power systems stabilizers through linear matrix inequalities. Paper presented at: Power Systems Conference and Exposition - PSCE'O6. Atlanta, GA, USA. https://doi.org/10.1109/ PSCE.2006.296280

Chilali M, Gahinet P, Apkarian P (1999) Robust pole placement in LMI regions. Siam 44(12): 2257-2270. https://doi. org/10.1109/9.811208

Cruz JJ (1996) Controle robusto multivariável. São Paulo, Brasil: Edusp.

Gahinet P, Nemirovskii A, Laub AJ, Chilali M (1994) The LMI control toolbox. Decision and Control, 1994., Proceedings of the $33^{\text {rd }}$ IEEE Conference Decision and Control, Lake Buena Vista, FL, USA (3): 2038-2041. https://doi.org/ 10.1109/CDC.1994.411440

Gu DW, Petkov PH, Konstantinov MM (2014) Robust control design with MATLAB ${ }^{\circledR}$. London: Springer Science \& Business Media.

Kaway F, Vinther K, Andersen P, Bendtsen JD (2017) An LMI approach for robust design of disturbance feedback control. Paper presented at: 56th Annual Conference of the Society of Instrument and Control Engineers of Japan (SICE). Kanazawa, Japan. https:// doi.org/10.23919/SICE.2017.8105449

Luo CC, Liu RF, Yang CD, Chang YH (2003) Helicopter Hocontrol design with robust flying quality. Aerospace Science and Technology 7(2): 159-169. https://doi.org/10.1016/S1270-9638(02)00012-3

Ogata K (2009) Modern control engineering. Upper Saddle River, NJ: Prentice Hall. $5^{\text {th }}$ edition.

Padfield GD (2008) Helicopter flight dynamics: the theory and application of flying qualities and simulation modelling. New Jersey, NJ, USA: John Wiley \& Sons.

Razzaghian A (2018) Robust Adaptive Neural Network Control of Miniature Unmanned Helicopter. Paper presented at: $26^{\text {th }}$ Iranian Conference on IEEE. https://doi.org/10.1109/ICEE.2018.8472637

Sakamoto T, Katayama H, Ichikawa A (2006) Attitude control of a helicopter model by robust PID controllers. Paper presented at: 2006 IEEE Conference on Computer Aided Control System Design, 2006 IEEE International Conference on Control Applications, 2006 IEEE International Symposium on Intelligent Control. Munich, Germany. https://doi.org/10.1109/CACSD-CCA-ISIC.2006.4776942

Scherer C, Gahinet P, Chilali M (1997) Multiobjective Output-Feedback. 42(7): 896-911. https://doi.org/ 10.1109/9.599969

Shen N, Su Z, Wang X, Li Y (2009) Robust controller design and hardware-in-loop simulation for a helicopter. Paper presented at: $4^{\text {th }}$ IEEE Conference on Industrial Electronics and Applications, ICIEA 2009. Xi'an, China. https://doi.org/10.1109/ICIEA.2009.5138789

Wang X, Lu G, Zhong Y (2013) Robust Hoattitude control of a laboratory helicopter. Robotics and Autonomous Systems 61(12): 12471257. https://doi.org/10.1016/j.robot.2013.09.006

Weilenmann MF, Christen U, Geering HP (1994) Robust Helicopter Position Control at Hover. Proceedings of the 1994 American Control Conference, Baltimore, MD, USA: 2491-2495

Yoneyama J, Kikuchi Y (2002) Robust control for uncertain systems with application to helicopter model. Proceedings of the 41st SICE Annual Conference SICE 2002 2(1): 1187-1190. https://doi.org/10.1109/SICE.2002.1195351 